\title{
An overview of deep learning in the field of dentistry
}

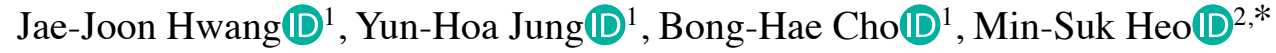 \\ ${ }^{1}$ Department of Oral and Maxillofacial Radiology, School of Dentistry, Pusan National University, Dental Research Institute, Yangsan, Korea \\ ${ }^{2}$ Department of Oral and Maxillofacial Radiology and Dental Research Institute, School of Dentistry, Seoul National University, Seoul, Korea
}

\section{ABSTRACT}

Purpose: Artificial intelligence (AI), represented by deep learning, can be used for real-life problems and is applied across all sectors of society including medical and dental field. The purpose of this study is to review articles about deep learning that were applied to the field of oral and maxillofacial radiology.

Materials and Methods: A systematic review was performed using Pubmed, Scopus, and IEEE explore databases to identify articles using deep learning in English literature. The variables from 25 articles included network architecture, number of training data, evaluation result, pros and cons, study object and imaging modality.

Results: Convolutional Neural network $(\mathrm{CNN})$ was used as a main network component. The number of published paper and training datasets tended to increase, dealing with various field of dentistry.

Conclusion: Dental public datasets need to be constructed and data standardization is necessary for clinical application of deep learning in dental field. (Imaging Sci Dent 2019; 49: 1-7)

KEY WORDS: Artificial Intelligence; Deep Learning; Dentistry; Radiology

\section{Introduction}

Artificial intelligence (AI) has evolved from the concept of strong AI, which imitates human intelligence, to the implementation of weak AI that can solve certain problems. ${ }^{1}$ Studies of weak AI explore ways to construct algorithms that can learn from data and make predictions. Machine learning is a branch of computer science that builds algorithms guided by data. ${ }^{2}$ Among them, neural networks (NNs), which consist of nodes and weights, were one of the first types of AI algorithms to be developed. The computational power of these networks relies on the quality and quantity of training data, which allow these networks to update the weights of the connections. Simple network structures with only a few layers are known as "shallow" learning neural networks, whereas network structures that employ numerous and large layers are referred to as "deep"

*This study was supported by 2017 Clinical Research Grant from Pusan National University Dental Hospital.

Received November 27, 2018; Revised December 15, 2018; Accepted December 17, 2018 *Correspondence to: Prof. Min-Suk Heo. Department of Oral and Maxillofacial Radiology, School of Dentistry, Seoul National University, 101 Daehak-ro, Jongno-gu, Seoul 03080, Korea

Tel) 82-2-2072-3016,E-mail) hmslsh@snu.ac.kr learning neural networks. ${ }^{3}$ Deep learning structures referred to as convolutional neural networks (CNNs), which can extract many features from abstracted layers of filters, are mainly used for processing large and complex images. Deep learning is being accelerated by the development of self-learning back-propagation algorithms that progressively refine the results from the data, as well as by increases in computational power. Due to these rapid technological advances, AI, represented by deep learning, can be used for real-life problems and is applied across all sectors of society. ${ }^{4}$ The diagnostic accuracy of deep learning algorithms in the medical field is approaching levels of human expertise, changing the role of computer-assisted diagnosis from a 'second-opinion' tool to a more collaborative one. ${ }^{3}$ The development of AI applications in the dental field is also remarkable. ${ }^{1,2}$ In this article, papers about deep learning applied to the field of oral and maxillofacial radiology will be reviewed.

\section{Materials and Methods}

\section{Search strategy}

In PubMed, Scopus, and the IEEE Xplore Digital Library, 
a search was performed for 'deep learning OR neural network' and 'dental AND (diagnosis OR detection OR classification OR segmentation)' extending through December 2018, and 144, 33, and 32 search results were obtained, respectively. A total of 25 peer-reviewed papers were obtained by removing articles not written in English, those focusing on non-dental fields, papers not related to imaging dentistry, as well as reviews, editorials, and in-press papers. The multilayer perceptron emerged as an early field of deep learning, and papers on this topic were excluded from this study because it is not a true end-to-end learning method-it learns features extracted from images using existing machine learning algorithms - and it has shallow networks and limited accuracy when the number of layers is increased. ${ }^{5}$

\section{Data extraction}

Study-specific data describing deep learning architecture, the size of the training datasets, evaluation results, advantages and disadvantages, the object of the study, and imaging modality were collected, in addition to other variables such as author and publication year.

\section{Results}

The data extracted from the selected papers are summarized in Table 1.

In all studies, $\mathrm{CNN}$ was used as a main network component, and there were also studies using various other types of networks, such as long short-term memory and siamese networks, in addition to CNNs. CNN-based papers have appeared in the field of dentistry since 2016, and subsequently, more and more dentistry papers using $\mathrm{CNN}$ have been published (Fig. 1).

The median size of the datasets used for training also tended to increase, from 100 units to 1000 units (Fig. 2).

Many papers that used pretrained networks such as Alexnet, VGG, GoogLeNet, and Inception v3 showed good results for general purposes. ${ }^{31}$ However, the structure of CNN networks tends to change from networks with shallow layers to deeper or problem-specific home-made or complex networks.

These studies dealt with various field of dentistry. Most of them were related to teeth, but other subjects such as the gingiva and periodontium, the dental arch, osteoporosis, and anatomical landmarks were also studied using deep learning (Table 2).

Various imaging modalities have been studied in conjunction with the abovementioned subjects. Efforts are underway to diagnose dental disease using traditional 2-di- mensional radiographs (intraoral and panoramic), as well as using 3-dimensional cone-beam computed tomography (CBCT). Other studies have investigated new modalities in dental applications, such as quantitative light-induced fluorescence, optical coherence tomography, and the use of intra-oral laser scanners.

\section{Discussion}

Computer assisted diagnosis (CAD) software in the medical field has been used to obtain second opinions, but the design and tuning of conventional CAD tends to be very arduous. Recently, deep learning techniques have been integrated into $\mathrm{CAD}$, with promising results for various medical applications. ${ }^{32,33}$ The qualitative and quantitative applications of deep learning in dentistry are also expanding, but certain areas need to be complemented to promote the continued development of deep learning research in oral and maxillofacial radiology.

However, because all the data sets used in the research analyzed herein were in-house, objective comparison of the studies was difficult. Only a single study tried to evaluate the accuracy of developed networks using other public datasets. ${ }^{23}$ Efforts are needed to develop a public dataset, such as in the medical field, ${ }^{34}$ to develop algorithms that can be used in clinical applications. In order to achieve this, researchers need to release the data used in their papers with appropriate removal of personal information, and legal and institutional support from each country is also necessary. ${ }^{35,36}$ There is also a need to build a common, free repository that can reliably collect, catalog, and archive publicly available data in the dental field.

The overall increase in the size of training datasets is desirable for clinical applications of deep learning to the dental field. However, most studies used relatively small data sets (fewer than 1000 units per group), and the accuracy of most studies was less than $90 \%$. This is below the clinically expected accuracy of $98 \%-99 \% .{ }^{37}$ Deep learning requires a large amount of data because it learns features directly from the data via an end-to-end process. In an anatomical classification study of CT data, at least 1,000 data sets per group were required to achieve $98 \%$ validation accuracy with deep learning, and 4,092 data sets per group were required to reach the desired accuracy of $99.5 \%{ }^{38}$ CBCT, which is the most popular 3D imaging modality in the dental field, does not utilize defined Hounsfield unit values like medical $\mathrm{CT}$, and the pixel values of the acquired images change at every exposure. ${ }^{39}$ The image quality and magnification of panoramic radiographs, which are com- 







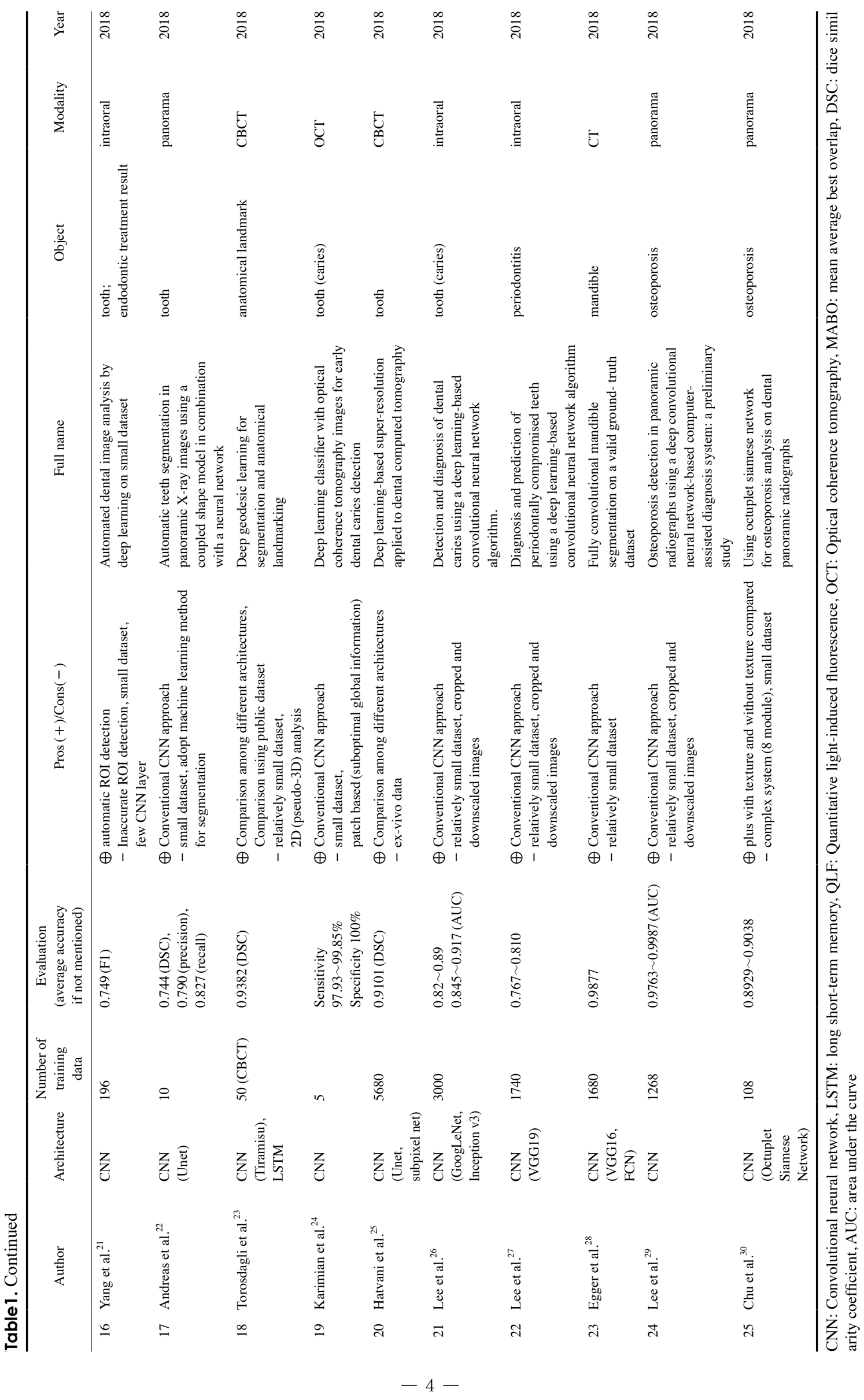




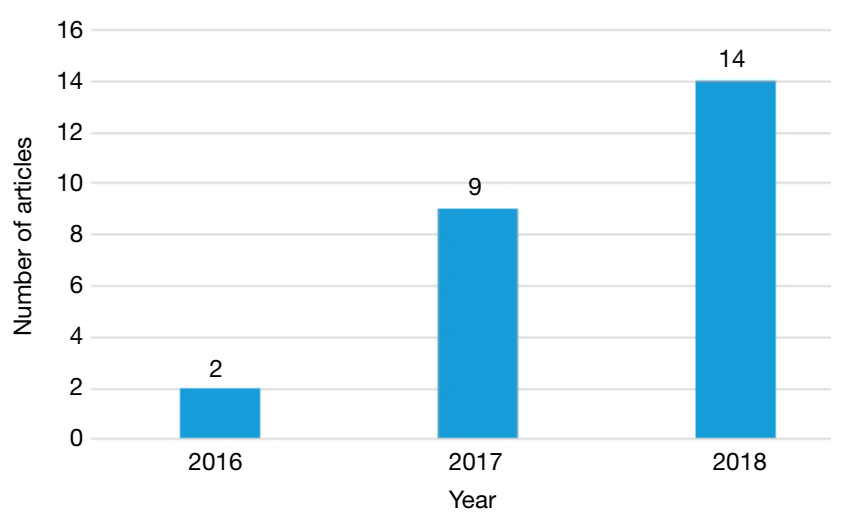

Fig. 1. Number of articles from 2016 to 2018.

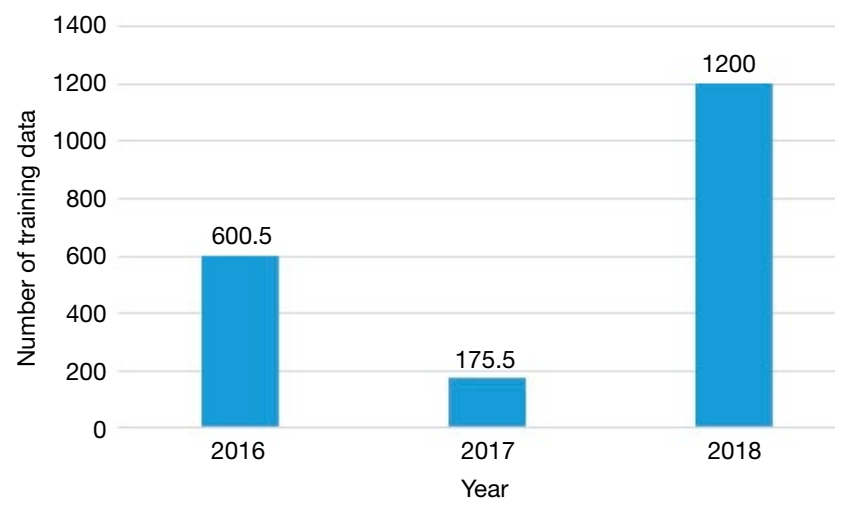

Fig. 2. Median size of training datasets from 2016 to 2018.

Table 2. Frequency of subjects in deep learning articles

\begin{tabular}{lc}
\hline \multicolumn{1}{c}{ Subject } & Frequency \\
\hline Tooth related & 12 \\
Dental plaque & 3 \\
Gingiva or periodontium & 2 \\
Osteoporosis & 2 \\
etc. & 5 \\
\hline
\end{tabular}

monly used in dental practice, depend on the positioning of the patient. ${ }^{40}$ Therefore, to achieve clinically meaningful high accuracy, trans-hospital or hybrid data sets from multiple machines and conditions are likely to be needed due to the nature of dental images. For this reason, it is especially important to emphasize the need to construct a largescale dental public dataset to make the clinical application of deep learning possible.

It is also necessary to emphasize the need for data standardization in the dental field, as well as for standardization of data set construction. In particular, CBCT exhibits large image variation according to brand, machine, and exposure conditions, which can be an obstacle to deep learning re- search. For example, collecting and learning data on a machine-by-machine basis is difficult because models learned on one machine do not apply to other machines. Although attempts have been made to develop guidelines in Europe, Germany, and England regarding the image quality of CBCT, no international standard has yet been established. ${ }^{41}$ Therefore, in order for 3-dimensional diagnosis using deep learning to be practical, an international standard for the quality of CBCT images needs to be established in the near future.

Many papers have used preprocessed images via manual cropping of the region of interest. This makes it difficult to analyze and compare results accurately due to errors in the manual process. Some papers ${ }^{9,10,19}$ have described networks that learned by dividing images into patches of a certain size. However, this method is limited because the network cannot learn the whole image, and instead only focuses on a small part of the image. Some papers ${ }^{21,22,24}$ used downsampling, which might delete important details of the image. These choices seem to have been made due to limitations in the amount of data or computational power, as indicated in the limitations sections of some papers. ${ }^{21,22}$ However, as computing power per cost increases, it is necessary to use entire images to learn, without any artificial manipulation in the preprocessing stage, in order to obtain more accurate and general results.

Currently, the use of AI is expanding in the medical field. For example, Watson, developed by IBM, has been used to support doctors' clinical decisions. ${ }^{42}$ However, the clinical accuracy of $\mathrm{AI}$ in the dental field must be verified with a variety of cases and imaging modalities due to the difficulty of standardizing dental radiology before AI can take on a more important role in making diagnostic recommendations. Furthermore, current AI algorithms function as black boxes, making it difficult for humans to identify or adjust the criteria used for diagnoses. ${ }^{43}$ Therefore, in order to increase the reliability of AI, it is necessary to develop a visualization and modification tool for deep learning networks that can be easily understood and edited by humans.

\section{References}

1. Park WJ, Park JB. History and application of artificial neural networks in dentistry. Eur J Dent 2018; 12: 594-601.

2. Mupparapu M, Wu CW, Chen YC. Artificial intelligence, machine learning, neural networks, and deep learning: futuristic concepts for new dental diagnosis. Quintessence Int 2018; 49: 687-8.

3. Burt JR, Torosdagli N, Khosravan N, RaviPrakash H, Mortazi A, Tissavirasingham F, et al. Deep learning beyond cats and dogs: recent advances in diagnosing breast cancer with deep neural 
networks. Br J Radiol 2018; 91: 20170545.

4. Rabuñal JR, Dorado J. Artificial neural networks in real-life applications. IGI Global: Hershey; 2005. p. 166-346.

5. Panchal G, Ganatra A, Kosta YP, Panchal D. Behaviour analysis of multilayer perceptrons with multiple hidden neurons and hidden layers. Int J Comput Theory Eng 2011; 3: 332-7.

6. Imangaliyev S, van der Veen MH, Volgenant CM, Keijser BJ, Crielaard W, Levin E. Deep learning for classification of dental plaque images. In: Conca PP, Nicosia GG. Machine learning, optimization, and Big data. Second International Workshop, MOD 2016, Volterra, Italy, August 26-29, 2016, Revised Selected Papers. Heidelberg: Springer. 2016. p. 407-10.

7. Eun H, Kim C. Oriented tooth localization for periapical dental $\mathrm{X}$-ray images via convolutional neural network. 2016 Asia-Pacific Signal and Information Processing Association Annual Summit and Conference (APSIPA 2016); 2016 Dec 13-16; Jeju, Korea. Red Hook, NY: IEEE; p. 33-9.

8. De Tobel J, Radesh P, Vandermeulen D, Thevissen PW. An automated technique to stage lower third molar development on panoramic radiographs for age estimation: a pilot study. $\mathrm{J}$ Forensic Odontostomatol 2017; 2: 42-54.

9. Rana A, Yauney G, Wong LC, Gupta O, Muftu A, Shah P. Automated segmentation of gingival diseases from oral images. IEEE-NIH 2017 Special Topics Conference on Healthcare Innovations and Point-of-Care Technologies. Bethesda, MD: National Institutes of Health. p. 144-7.

10. Lee H, Park M, Kim J. Cephalometric landmark detection in dental X-ray images using convolutional neural networks. Proc. SPIE 10134, Medical Imaging 2017: Computer-Aided Diagnosis, 101341W (3 March 2017). Available from: https://doi. org/10.1117/12.2255870.

11. Prajapati SA, Nagaraj R, Mitra S. Classification of dental diseases using CNN and transfer learning. 2017 5th International Symposium on Computational and Business Intelligence (ISCBI), Dubai, 2017, p. 70-4. Available from: https://doi. org/10.1109/ISCBI.2017.8053547.

12. Imangaliyev $\mathrm{S}$, van der Veen $\mathrm{MH}$, Volgenant CM, Loos BG, Keijser BJ, Crielaard W, et al. Classification of quantitative light-induced fluorescence images using convolutional neural network. arXiv:1705.09193. 2017 [cited 2018 November 20]. Available from: https://arxiv.org/pdf/1705.09193.pdf

13. Miki Y, Muramatsu C, Hayashi T, Zhou X, Hara T, Katsumata A, et al. Classification of teeth in cone-beam CT using deep convolutional neural network. Comput Biol Med 2017; 80: 24-9.

14. Yauney G, AngelinoK, Edlund DA, Shah P. Convolutional neural network for combined classification of fluorescent biomarkers and expert annotations using white light images. 2017 IEEE 17th International Conference on Bioinformatics and Bioengineering (BIBE), Washington, DC, 2017, p. 303-9. Available from: https://doi.org/10.1109/BIBE.2017.00-37

15. Oktay AB. Tooth detection with Convolutional Neural Networks. 2017 Medical Technologies National Congress (TIPTEKNO), Trabzon, 2017, p. 1-4. Available from: https://doi. org/10.1109/TIPTEKNO.2017.8238075

16. Miki Y, Muramatsu C, Hayashi T, Zhou X, Hara T, Katsumata A, et al. Tooth labeling in cone-beam CT using deep convolutional neural network for forensic identification. In: Armato SG, Petrick NA. Medical Imaging 2017: Computer-Aided Diagnosis.
Proceedings Volume 10134, SPIE Medical Imaging; 2017 Feb 11-16; Orlando, USA: SPIE; 2017. Available from: https://doi. org/10.1117/12.2254332

17. Murata S, Lee C, Tanikawa C, Date S. Towards a fully automated diagnostic system for orthodontic treatment in dentistry. 2017 IEEE 13th International Conference on e-Science (e-Science), Auckland, 2017. p. 1-8. Available from: https://doi. org/10.1109/eScience.2017.12

18. Xu X, Liu C, Zheng Y. 3D tooth segmentation and labeling using deep convolutional neural networks. IEEE Trans Vis Comput Graph (in press).

19. Du X, Chen Y, Zhao J, Xi Y. A convolutional neural network based auto-positioning method for dental arch in rotational panoramic radiography. Conf Proc IEEE Eng Med Biol Soc 2018; 2018: 2615-8.

20. Zhang K, Wu J, Chen H, Lyu P. An effective teeth recognition method using label tree with cascade network structure. Comput Med Imaging Graph 2018; 68: 61-70.

21. Yang J, Xie Y, Liu L, Xia B, Cao Z, Guo C. Automated dental image analysis by deep learning on small dataset. 2018 IEEE 42nd Annual Computer Software and Applications Conference (COMPSAC), Tokyo, 2018. 492-7. Available from: https://doi. org/10.1109/COMPSAC.2018.00076

22. Andreas W, Sudesh G, Stefan W. Automatic teeth segmentation in panoramic X-ray images using a coupled shape model in combination with a neural network. Proceedings from the 21st International Conference on Medical Image Computing and Computer Assisted Intervention, Part IV; 2018 Sep 16-20; Granada, Spain. Basel: Springer; 2018. Available from: https:// doi.org/10.1007/978-3-030-00937-3_81.

23. Torosdagli N, Liberton DK, Verma P, Sincan M, Lee JS, Bagci U. Deep geodesic learning for segmentation and anatomical landmarking. IEEE Trans Med Imaging (in press).

24. Karimian N, Salehi HS, Mahdian M, Alnajjar H, Tadinada A. Deep learning classifier with optical coherence tomography images for early dental caries detection. Proc. SPIE 10473, Lasers in Dentistry XXIV, 1047304 (8 February 2018); Available from: https://doi.org/10.1117/12.2291088

25. Hatvani J, Horváth A, Michetti J, Basarab A, Kouamé D, Gyöngy M. Deep learning-based super-resolution applied to dental computed tomography. IEEE Transactions on Radiation and Plasma Medical Sciences. Available from: https://doi. org/10.1109/TRPMS.2018.2827239

26. Lee JH, Kim DH, Jeong SN, Choi SH. Detection and diagnosis of dental caries using a deep learning-based convolutional neural network algorithm. J Dent 2018; 77: 106-111.

27. Lee JH, Kim DH, Jeong SN, Choi SH. Diagnosis and prediction of periodontally compromised teeth using a deep learning-based convolutional neural network algorithm. J Periodontal Implant Sci 2018; 48: 114-23.

28. Egger J, Pfarrkirchner B, Gsaxner C, Lindner L, Schmalstieg D, Wallner J. Fully convolutional mandible segmentation on a valid ground - truth dataset. Conf Proc IEEE Eng Med Biol Soc 2018; 2018: 656-60.

29. Lee JS, Adhikari S, Liu L, Jeong HG, Kim H, Yoon SJ. Osteoporosis detection in panoramic radiographs using a deep convolutional neural network-based computer-assisted diagnosis system: a preliminary study. Dentomaxillofac Radiol 2018: 
20170344 (in press).

30. Chu P, Bo C, Liang X, Yang J, Megalooikonomou V, Yang F, et al. Using octuplet siamese network for osteoporosis analysis on dental panoramic radiographs. Conf Proc IEEE Eng Med Biol Soc 2018; 2018: 2579-82.

31. Kim YD, Jang TW, Han BY, Choi, SJ. Learning to select pretrained deep representations with bayesian evidence framework. 2016 IEEE Conference on Computer Vision and Pattern Recognition (CVPR), Las Vegas, NV, 2016, pp. 5318-5326. p. 531826. Available from: https://doi.org/10.1109/CVPR.2016.574

32. Cheng JZ, Ni D, Chou YH, Qin J, Tiu CM, Chang YC, et al. Computer-aided diagnosis with deep learning architecture: applications to breast lesions in US images and pulmonary nodules in CT scans. Sci Rep 2016; 6: 24454.

33. Suzuki K. Overview of deep learning in medical imaging. Radiol Phys Technol 2017; 10: 257-73.

34. Greenspan H, Ginneken BV, Summers RM. Guest editorial: deep learning in medical imaging: overview and future promise of an exciting new technique. IEEE Trans Med Imaging 2016; 35: 1153-9.

35. Berman JJ. Confidentiality issues for medical data miners. Artif Intell Med 2002; 26: 25-36.

36. Cooper T, Collman J. Managing information security and privacy in healthcare data mining. In: Chen H, Fuller SS, Friedman $\mathrm{CH}$. Medical informatics: knowledge management and data mining in biomedicine. New York: Springer; 2005. p. 95-137.
37. Weese J, Lorenz C. Four challenges in medical image analysis from an industrial perspective. Med Image Anal 2016; 33: 44-9.

38. Cho J, Lee K, Shin E, Choy G, Do S. How much data is needed to train a medical image deep learning system to achieve necessary high accuracy? arXiv:1511.06348 [Preprint]. 2016 [cited 2018 Nov 20] Available from: https://arxiv.org/abs/1511.06348

39. Pauwels R, Araki K, Siewerdsen JH, Thongvigitmanee SS. Technical aspects of dental CBCT: state of the art. Dentomaxillofac Radiol 2015; 44: 20140224.

40. Devlin H, Yuan J. Object position and image magnification in dental panoramic radiography: a theoretical analysis. Dentomaxillofac Radiol 2013; 42: 29951683.

41. de Las Heras Gala H, Torresin A, Dasu A, Rampado O, Delis H, Hernández Girón I, et al. Quality control in cone-beam computed tomography (CBCT) EFOMP-ESTRO-IAEA protocol (summary report). Phys Med 2017; 39: 67-72.

42. Chen Y, Elenee Argentinis JD, Weber G. IBM Watson: how cognitive computing can be applied to big data challenges in life sciences research. Clin Ther 2016; 38: 688-701.

43. Anifowose FA. Artificial intelligence application in reservoir characterization and modeling: whitening the black Box. Proceedings of the SPE Saudi Arabia section Young Professionals Technical Symposium; 2011 Mar 14-16; Dharan, Saudi Arabia. Dharan: Society of Petroleum Engineers; 2011. Available from: https://doi.org/10.2118/155413-MS.v 